\title{
Save your Boat from Sinking Earlier: Don't be Hurry for Direct Laryngoscopic Assessment in a Dysphagia Patient
}

\author{
${ }^{1}$ Tareq Mohammad MBBS, FCPS, \\ Assistant Registrar ENT, \\ Bangladesh Medical College \\ Hospital
}

\author{
${ }^{4}$ ASM Lutfur Rahman MBBS, \\ MCPS, DLO, MRCPS (Glasgow), \\ Resident Surgeon, \\ Bangladesh Medical College \\ Hospital
}

${ }^{7}$ Towsif Bin Mamoon, MBBS, Assistant Registrar, ENT, Bangladesh Medical College Hospital

\begin{abstract}
Myasthenia gravis (MG) is a relatively rare autoimmune disorder caused by an antibody-mediated blockade of neuromuscular transmission resulting in skeletal muscle weakness. The autoimmune attack occurs when autoantibodies form against the nicotinic acetylcholine postsynaptic receptors at the neuromuscular junction of skeletal muscles. Patients with myasthenia, usually presents with painless, specific muscle weakness. Typically it affects extra ocular, bulbar, or proximal limb muscles. Approximately 50\% to $80 \%$ patients presents with ocular and $20 \%$ patient presents with bulbar symptoms. Though some specific clinical and serological tests are there to confirm the diagnosis, a high index of suspicion and detail clinical history brings the utmost importance. In this article we highlighted 02 cases of MG; a boy of 15 years and a male farmer 42 year presented with the presentation of MG.
\end{abstract}

Keywords:- MG, dysphagia, direct laryngoscopy.

\section{INTRODUCTION}

Dysphagia is one of the most common outdoor presentation by a patient to an ENT surgeon. In a busy clinic sometime we miss proper evaluation of this patient especially when it is presented by younger group of patients. Sometimes we admit our patients for evaluation especially by Direct laryngoscopic assessment when other relevant investigation shows negative result. But it should be remembered that it can be the tip of iceberg. Dysphagia can be a sole symptom by a Myasthenia patient to the ENT clinic.

\author{
${ }^{3}$ Nazmul Hossain Chowdhury, \\ MBBS, FCPS, \\ Assistant Professor, ENT, \\ Bangladesh Medical College \\ Hospital
}

${ }^{6}$ Farid Uddin Milki, MBBS, DLO, Consultant ENT, Bangladesh Medical College Hospital

\author{
${ }^{8}$ Md. Ashraful Islam MBBS, FCPS, FICS, FRCS \\ (Glasgow), Professor and Head, \\ Otolaryngology-Head \& Neck Surgery, \\ Bangladesh Medical College Hospital
}

Myasthenia gravis (MG) is a relatively rare autoimmune disorder caused by an antibody-mediated blockade of neuromuscular transmission resulting in skeletal muscle weakness ${ }^{1}$. The autoimmune attack occurs when autoantibodies form against the nicotinic acetylcholine postsynaptic receptors at the neuromuscular junction of skeletal muscles ${ }^{2}$.Patients with myasthenia, usually presents with painless, specific muscle weakness. Typically affects extraocular, bulbar, or proximal limb muscles. In approximately $50 \%$ to $80 \%$ patients presents with ocular symptoms ${ }^{3}$. Approximately $20 \%$ patient presents with bulbar symptoms ${ }^{3}$. Though some specific clinical and serological tests are there to confirm the diagnosis, a high index of suspicion and detail clinical history brings the utmost importance.

Though in such cases any sort of surgery under general anesthesia might lead to pay a lot of sufferings for the surgeon and anesthetist. Here we present 2 cases who were admitted in ENT department from a busy OPD for $\mathrm{D} / \mathrm{L}$ assessment due to dysphagia.

\section{CASE REPORT}

\section{Case 1:}

A 15 years old boy, $4^{\text {th }}$ issue of his nonconsanguineous parents, immunized as per EPI schedule, presented with nasal voice and dysphagia for last 6 months. He also complaints bilateral partial dropping of both eyes and easy fatigability during prolonged walking and running for same duration. All these symptoms more marked in evening but full recovery by the following morning. He also developed double vision for same duration. There was no history of cough, dyspnea, dysarthria, joint pain, fever or skin rash. On examination he 
was conscious, bilateral partial ptosis of both eyes, vitals were within normal limit. Developmentally age appropriate. On nervous system examination, eye movement was limited in upward direction, visual acuity, visual field, pupillary light reflex and accommodation reflex were normal. On motor examination, there was no muscle wasting, tenderness or fasciculation. Muscle tone was normal, power was diminished on both lower limbs and deep tendon reflex was normal. Fatigability test, neostigmine test and ice pack test were positive (fig 1). Other systemic examination revealed normal findings.

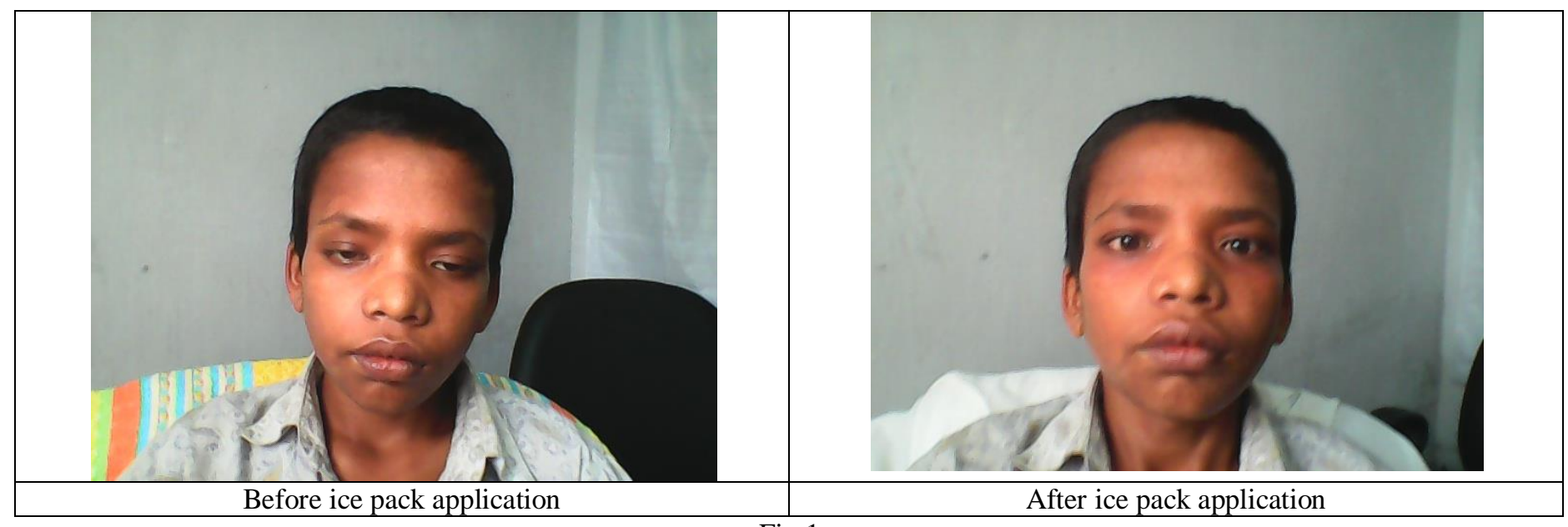

Fig 1

All his routine examination including $\mathrm{CBC}$, renal function, serum electrolytes, CXR, FOL were normal. EMG - Normal, NCV - Normal, Single fiber repetitive nerve stimulation test revealed significant decremental response between $1^{\text {st }}$ and $4^{\text {th }}$ stimulation. CT scan of chest showed normal finding. So, we diagnosed this case as Juvenile Myasthenia Gravis.so, the plan of D/L assessment under G/A was abounded.

\section{$>$ Case 2:}

A 42 years male farmer was admitted to ENT department from our busy OPD as a case of Dysphagia for evaluation. Patient presented with progressive dysphagia for 6 months and dysarthria for 8 months. His dysphagia started 6 months back from when he is noticing difficulty in swallowing both for solid and liquid specially at evening and more at night. He gets some relief during breakfast and lunch. He also mentioned difficulty in speaking for same duration. He feels slight slurring at morning but it worsens with time. On further query he mentioned that he also has limb weakness for 6 years for what he consulted several quack doctors of his village but never consulted to any certified quality doctors as it was not bothered him too much. As his weakness worsens with time at afternoon, he accepted it as normal weakness after day time work load.

On general examination he was anxious but cooperative, vitals were within normal limit. His body built was below average, body weight $45 \mathrm{~kg}$ and height of 5.2 feet. On local examination he had bilateral ptosis, intraoral examination revealed normal oral cavity and oropharynx but weakness noted on soft palatal movement. Indirect laryngoscopic examination was also normal. On extraoral examination there was weakness on masticator muscles on repeated attempt to examine the power of masticator muscle specially masseter and temporalis on clinching. On examination of neck there were no abnormality. On motor examination, there was no muscle wasting, tenderness or fasciculation. Muscle tone was normal, power was diminished on both lower limbs and deep tendon reflex was normal. Fatigability test, neostigmine test and ice pack test were positive. Other systemic examination revealed normal findings. Fiber optic laryngoscopy, upper GI endoscopy was also normal. On single fiber repetitive nerve stimulation test, the decremental response was maximum at $4^{\text {th }}$ and $5^{\text {th }}$ stimulation and then a plateau was noted. $\left(1^{\text {st }}\right.$ response at $8.32 \mathrm{mv}$ and it progressively decreased on $4^{\text {th }}$ and $5^{\text {th }}$ stimulation with peak amplitude was 4.83 and $4.76 \mathrm{mv})$. EMG was normal. His other investigation like CXR, CBC, CT chest and neck was normal. So, with all of these this patient was diagnosed as a case of Myasthenia gravis, and his plan of $\mathrm{D} / \mathrm{L}$ assessment was essentially avoided. Patient was discharged after counselling about the disease process

\section{DISCUSSION}

Myasthenia gravis is a autoimmune disease of the neuromuscular junction characterized by the formation antiacetylcholine receptor antibodies (anti-AchR abs) that blocks neuromuscular transmission, leads to skeletal muscle weakness. Weakness in myasthenia is due to impaired action potential propagation caused by damage to post-synaptic acetylcholine receptors due to which muscles do not depolarize ${ }^{4}$.Skeletal muscles in ocular and facial distribution are commonly affected and typical presentation is with ptosis and diplopia ${ }^{5}$. Others symptoms are dysphagia, dysarthria, progressive limb weakness etc. It should be kept in mind that dysphagia may be the sole symptom $^{6}$ in few cases. Dysphagia occurs due to involvement of the pharyngeal and striated esophageal muscles, is seen in $30-60 \%$ cases and in $15 \%$ can be the only symptom ${ }^{7}$. 
Dysphagia is one of the common presentations by a patient to ENT specialist. It is a trend by a physician to advice Upper GI endoscopy followed by D/L assessment under G/A to establish the cause. A patient of myasthenia can also present with the similar problem and if the physician fails to diagnose the patient before going for $\mathrm{D} / \mathrm{L}$ assessment, it can be a grave for that surgeon and anesthetist.

General anaesthesia with traditional muscle relaxant for any sort of surgery in a myasthenic patient can cause significant risk of death or other serious complication. The risk is mainly due to a markedly higher sensitivity to muscle relaxants observed in this group of patients, even in period of complete remission ${ }^{8}$. Myesthenia patients may take a longer reversal time and sometimes may require prolonged ventilation in ICU setting ${ }^{9}$. A significant, though less common, perioperative risk in myasthenic patient is cholinergic crisis where the patient may develop respiratory failure or delayed postoperative extubation for more than 24 hours resulting from myasthenic weakness.

The most proper approach during anaesthesia in patients with myasthenia gravis is to apply deep inhalational anaesthesia without muscle relaxing agents, however in head neck surgeries this practice is quite difficult. Though many anaesthesiologist routinely decided against using muscle relaxants in such clinical cases, some of them are trying to use some alternates.

The main aim of this case report is to highlight the importance of adequate preoperative evaluation to avoid per-operative and postoperative hazards and unexpected morbidity and mortality as well.

\section{CONCLUSION}

Myasthenia gravis is the most common neurological disorder. It is easier to diagnose by a neurologist or medicine consultant but to an ENT consultant, patient may present with dysphagia as a sole symptom. So, a high index of suspicion is very important to diagnose this patient before going any sort of surgery under general anesthesia. Avoidance of drugs associated with worsening of myasthenic weakness is important to prevent iatrogenic worsening that can precipitate myasthenic crisis which has high morbidity and mortality rates.

\section{REFERENCES}

[1]. Strauss AJL, Seigal BC, Hsu KC. Immunofluorescence demonstration of a muscle binding complement fixing serum globulin fraction in Myasthenia Gravis. Proc Soc Exp Biol. 1960. 105:184.

[2]. Patric J, Lindstrom JM. Autoimmune response to acetylcholine receptor. Science. 1973. 180:871.

[3]. Patric J, Lindstrom JM. Autoimmune response to acetylcholine receptor. Science. 1973. 180:871.

[4]. Phillips LH. Ann N Y. The epidemiology of myasthenia gravis. Acad Sci. 2003;998:407-412.
[5]. Tibbling L, Gustafsson B. Dysphagia and its consequences in the elderly. Dysphagia 1991;6:200.

[6]. Jagpal S, Yogita M, Nikhil K. Myasthenia gravis masquerading as dysphagia: unveiled by magnesium infusion.BMJ case rep> v.2014:2014 > PMC3992550

[7]. Sanders DB, Howard JF. Disorders of neuromuscular transmission. In: Bradley WG, Daroff RB, Fenichel $\mathrm{GM}$, et al. eds. Neurology in clinical practice. vol 2 1st edn Massachusetts: Butterworth, Heinemann, 1991:1819

[8]. Dillon FX. Anesthesia issues in the perioperative management of myasthenia gravis. Semin Neurol. 2004;24:83-94

[9]. Heliopoulos I, Patlakas G, Vadikolias K, et al. Maximal voluntary ventilation in myasthenia gravis. Muscle Nerve. 2003;27:715-9. 